\title{
ETNOGRAFIA: ESTEATÉGIA METODOLÓGICA UTILIZADA PARA CONTEXTUALIZAR O \\ CENÁRIO CULTURAL DO CTI DE UM HOSPITAL UNIVERSITÁRIO*
}

\section{ETNOGRAPHY: METHODOLOGICAL STRATEGER FOR CONTEXTURE OF A CULTURAL BACKGROUND OF A UNIVERSITY HOSPITAL ICC}

\begin{abstract}
BASTOS, MAR. Estratégia metodológica utilizada para contextualizar o cenário cultural do CTI de um hospital universitário. Rev Esc Enf USP, v. 35, n. 2, p. 163-71, jun. 2001.

RESUMO

O presente trabalho é parte de um estudo etnográfico realizado em um CTI de um hospital universitário que teve o objetivo de descrever a cultura dos intensivistas. São apresentados dominios e taxonomias culturais elaborados a partir dos relatos dos intensivistas. Os resultados aqui apresentados demostraram que a etnografia é uma estratégia metodológica adequada para contextualização do cenário cultural sob o ponto de vista de quem vivencia o cotidiano do trabalho na terapia intensiva.
\end{abstract}

PALAVRAS-CHAVE: Etnografia. Cultura organizacional. Unidades de Terapia Intensiva.

\begin{abstract}
This work is a part of an ethnographic study accomplished in a university hospital ICC that had as objective to describe the intensive care workers 'culture. It is presented cultural domains a taxonomies developed from the workers' narration The results here presented show that ethnography is a methodological strategy appropriate for a cultural background contexture from a viewpoint of those who experiment the everyday intensive care work.
\end{abstract}

KEYWORDS: Ethnography. Organizational culture. Intensive care center.

\section{INTRODUÇÃO}

A concepção de Centro de Tratamento Intensivo CTI surgiu no ano de 1799, durante a campanha de Napoleão Bonaparte no Egito, quando foram idealizadas ambulâncias móveis com recursos materiais e humanos necessários ao atendimento dos feridos graves dos campos de batalhais ${ }^{(1)}$.

As unidades de Terapia Intensiva surgem nos anos 50 caracterizadas como um "sistema tecnológico de instrumentos, conhecimentos e habilidades" destinado à assistência ao indivíduo criticamente enfermo. As primeiras iniciativas de observação contínua a pacientes, surgiram nos anos 30, nas chamadas salas de recuperação pós-anestésica. $\mathrm{Na}$ década de 40 e início dos anos 50 surgem os primeiros esforços no sentido de agrupar pacientes instáveis, exigindo uma concentração de profissionais e equipamentos ${ }^{(2)}$

Dois fatores contribuíram, fundamentalmente, para a consolidação dos Centros de Tratamento Intensivo: o número crescente de hospitalização de pacientes e o aumento de doenças complexas. Tal situação acentuou as dificuldades de assistência, tanto sob o ponto de vista dos recursos materiais e humanos disponíveis, quanto de recursos físicos; levando à restruturação dos hospitais para atender à nova demande ${ }^{(2)}$.

\footnotetext{
Projeto financiado pela FAPEMIG

** Doutor. Prof. Adjunto do Departamento de Enfermagem Básica da Escola de Enfermagem da UFMG.
}

marisa@enf.ufmg.br 
Com o mesmo objetivo de centralizar recursos num local específico do hospital para o atendimento de pacientes recuperáveis, os Centros de Tratamento Intensivo surgem no Brasil na década de $70^{(3)}$.

A reorganização da estrutura hospitalar com a concentração de pessoal treinado, de recursos materiais e tecnológicos em determinada área do hospital surge com o conceito de Cuidado Progressivo de Pacientes. Segundo esse modelo de organização hospitalar, os pacientes são agrupados de acordo com a gravidade de sua doença e dependência dos serviços de enfermagem ${ }^{(4)}$.

Assim, os Centros de Tratamento Intensivo são unidades integrantes dos hospitais que se destinam à assistência a pacientes criticamente enfermos. Nesses setores são concentrados recursos humanos qualificados, bem como recursos tecnológicos altamente sofisticados que possibilitam racionalização, rapidez e eficiência no trabalho.

Os trabalhos com temáticas relacionadas à Terapia Intensiva são, em grande parte, estudos quantitativos e que analisam procedimentos diagnóstico - terapêuticos de pacientes internados naquele setor.

$\mathrm{Na}$ revisão da literatura realizada, foi identificado que alguns autores tratam o CTI na sua dimensão cultural, numa perspectiva etnográfica, identificando significados, símbolos, valores, mitos e ritos compartilhados por pacientes ou profiss sionais $\left(5^{\prime} 6^{\prime} 7^{\prime} 8,9\right)$

Logo que comecei a trabalhar na Terapia Intensiva, ainda como estudante, despertava-me a atenção as peculiaridades do setor e dos profissionais de saúde que ali atuavam. Algumas atitudes e características os diferenciavam dos demais, pois observava a existência de uma identidade compartilhada entre os membros da Terapia Intensiva, identidade esta constituída por valores, idéias e significados.

Ao cursar o doutorado, tive a oportunidade de estudar o CTI e seus membros sob o ponto de vista cultural, descrevendo seus valores, mitos e estratégias de sobrevivência.

Assim foi realizada uma pesquisa etnográfica no CTI de um hospital universitário que teve como objetivo descrever a cultura dos intensivistas (10).

O presente artigo tem como objetivo relatar parte da tese desenvolvida, apresentando os dados relacionados à contextualização do cenário cultural do CTI, apontando para a adequação da etnografia enquanto estratégia metodológica para caracterizar o ambiente cultural, a partir dos relatos dos seus membros.
A apresentação desses dados está justificada pelo fato de que o método etnográfico, habitualmente utilizado para descrever culturas (objetivo do trabalho que deu origem a este artigo), mostra-se, também, adequado para a caracterização do cenário cultural.

Assim, foram descritos aspectos administrativos, conceituais e históricos do CTI a partir da interpretação dos relatos dos intensivistas, constituindo-se, portanto, em parte do conhecimento cultural compartilhado por eles.

\section{A OPÇÃO METODOLÓGICA}

Optei por realizar um estudo etnográfico uma vez que é adequado para compreender a complexidade dos fenômenos culturais da organização, sob a perspectiva de seus próprios membros, nos seus "próprios termos")"11.

A etnografia é o trabalho de descrição de uma cultura a partir da visão de mundo do nativo dessa cultura. Assim, a etnografia busca a compreensão do significado de ações e eventos para os participantes, significados esses expressos através da linguagem ou indiretamente através de ações, constituindo-se num sistema de significados complexos. Esse sistema de significados constitui a cultura. A etnografia é situada como uma estratégia teórico-metodológica para descobrir os simbolos de uma cultura e compreender suas relações dentro de um complexo sistema de significados ${ }^{(12)}$.

$\mathrm{Na}$ pesquisa realizada foram utilizadas a observação participante, a analise documental e a entrevista etnográfica como técnicas de coleta de dados (10).

Para compreender os universos simbólicos compartilhados pelos membros do CTI foram realizadas análise de domínio, taxonômica e temática ${ }^{(12)}$.

Da mesma forma, buscando compreender o contexto cultural no qual foi realizada a pesquisa sob o ponto de vista de quem o vivencia, foram, também, realizadas análise de domínio, taxonômica e temática (12).

A análise etnográfica é a busca da estrutura interna de uma cultura, suas partes e as relações entre essas partes, sob o ponto de vista dos nativos dessa cultura, denominados de informantes. Seu objetivo principal é a descoberta de "significados culturais".

A análise de domínio é o primeiro tipo de análise etnográfica. Seu objetivo é identificar categorias de significados culturais ${ }^{(12)}$.

Os domínios culturais são constituídos de três elementos básicos: termos inclusos (denominação das categorias menores); termos encobertos (nome dos domínios) e relação semântica entre os termos. Da 
análise de domínio surgem questões estruturais que visam compreender a organização cultural (12).

Após a identificação dos dominios culturais, dada a complexidade e necessidade de grande tempo de pesquisa, o pesquisador poderá focalizar alguns aspectos da cultura em estudo sem, no entanto, perder a visão do todo, denominado de "foco etnográfico"(12)

A partir daí, o pesquisador inicia uma investigação profunda dos vários dominios selecionados, buscando a sua estrutura de organização. Esta é a análise taxonômica dos dados etnográficos. A taxonomia estabelece a relação entre os termos inclusos de um domínio cultural, mostrando a sua organização interna, gerando classificações (12).

Uma visão mais ampla da cultura é obtida através da análise temática. Os temas culturais são definidos como "principios cognitivos recorrentes num determinado número de dominios, tácitos ou explícitos, mostrando o relacionamento entre os subsistemas de significado cultural"(12)

Considerando que a descrição do cenário cultural da terapia intensiva é parte fundamental para compreensão da prática dos intensivistas, foram construídos domínios e taxonomias relacionados ao contexto do setor a partir dos relatos dos informante ${ }^{(12)}$

Assim, a analise etnográfica foi, também, utilizada como estratégia metodológica para descrição do cenário cultural, ao invés da utilização, apenas, do resgate histórico documental e das observações do pesquisador, estratégias habitualmente utilizadas para descrição do contexto onde é realizada a pesquisa etnográfica.

\section{A Coleta de dados}

As primeiras observações tiveram a finalidade principal de contextualizar o cenário cultural no qual foi desenvolvido o trabalho, bem como de estabelecer o "rapport" entre aquelas pessoas que vivenciavam aquele cotidiano.

As fases da observação participante constituíram-se, na prática, em três fases principais, ou seja, $1^{a}$ fase: observação sem participação; $2^{\mathrm{a}}$ fase: observação com pequena participação e $3^{a}$ fase: observação com participação moderada. No entanto, é importante relatar que não foi possivel identificar uma nítida delimitação entre estas fases.

Foram identificadas algumas peculiaridades em se fazer observação participante numa unidade de assistência a pacientes graves, como, por exemplo, a dificuldade em observar atividades assistenciais sem nenhum tipo de envolvimento com o paciente ou com o profissional que prestava assistência.
Os registros etnográficos decorrentes da observação participante foram de natureza descritiva e reflexiva. As descrições foram relativas ao ambiente físico, comportamentos, ações, atividades específicas do setor e diálogos.

Os registros de natureza reflexiva foram relativos às minhas reflexões quanto a questões metodológicas ou teóricas que surgiram no decorrer da pesquisa e que necessitavam de esclarecimento ou elaboração de questões descritivas ou estruturais.

\section{A 1a fase: observação sem participação}

As atividades de observação sem nenhuma participação ocorreram apenas nas primeiras 36 horas de observação, quando fui apresentada aos membros do CTI e iniciei os primeiros registros de natureza mais descritiva, relativos aos recursos físicos humanos e materiais daquela unidade.

Nesta fase ocorreram conversas informais, com perguntas descritivas bem amplas e gerais que, na verdade, tinham o objetivo de criar um ambiente amistoso entre nós e contextualizar o cenário cultural em estudo. Alguns desses membros do CTI participaram da pesquisa tornando-se informantes, dentre eles, oito são enfermeiros, um é fisioterapeuta, um é auxiliar de enfermagem e dois são médicos.

$\mathrm{Na} 1$ a fase foram realizadas três entrevistas etnográficas com dois informantes, dos quais uma tornou-se a minha informante-chave. Informante chave é o membro que tem grande conhecimento da cultura estudada e que compreende o papel do pesquisador enquanto um aprendiz cultural(12).

\section{A $2^{\text {a }}$ fase: observação com pequena participação}

Ao iniciar as observações de atividades assistenciais compreendi que não poderia deixar de intervir nas situações, por mim identificadas, que expunham os pacientes a algum tipo de risco ou grande desconforto fisico. Assim, foram estabelecidos os critérios de participação, ou seja, a participação do pesquisador nas atividades assistenciais se deu apenas naquelas situações identificadas como de risco ou de desconforto para o paciente, situações estas que demandavam a intervenção da pesquisadora no ambiente.

Nesta fase, as observações tornaram-se mais seletivas a partir do momento em que os informantes indicaram, através de comportamentos e linguagem, aquilo que veio a se constituir no foco etnográfico da pesquisa - o processo de socialização dos novos membros.

Nesta fase realizei seis entrevistas etnográficas num processo concomitante de coleta e análise de dados. 


\section{A $3^{\text {a }}$ fase: observação com participação moderada}

Estabeleci como início da terceira fase o momento em que as observações de atividades assistenciais tornaram-se mais freqüentes. A partir desse momento, foi cada vez mais difícil uma participação apenas dentro dos critérios estabelecidos na segunda fase., uma vez que, nas observações feitas nas unidades com pacientes era dificil o não envolvimento nas atividades assistenciais, tendo em vista a necessidade constante de ajuda mútua entre os elementos da equipe de enfermagem.

Esta fase foi, também, de muitas reflexões. Reflexões relativas às estratégias metodológicas para validação das taxonomias e hipóteses temáticas que surgiam, reflexões sobre a possibilidade de aprofundar outros domínios que emergiam, reflexões sobre como e quando sair daquela cena cultural.

Pautada nas premissas do método etnográfico, a decisão de parar foi concomitante ao processo de saturação dos dados. A partir do momento em que os dados relativos aos domínios selecionados para aprofundamento foram ficando repetitivos, compreendi que era o momento de interromper a coleta de dados através da observação participante, embora outros domínios fossem emergindo das entrevistas etnográficas. No entanto, a decisão de parar foi consolidada frente à necessidade de cumprir prazos no curso de pós-graduação, como também, a compreensão da impossibilidade de aprender aquela subcultura em toda a sua complexidade, por mais tempo que permanecesse ali.

Nesta fase foram realizadas cinco entrevistas etnográficas.

\section{RESULTADOS: CONTEXTUALIZANDO $O$ CENÁRIO CULTURAL}

Considerando que o presente artigo tem o objetivo de apresentar parte da pesquisa realizada, ou seja, os resultados relativos à contextualização do cenário cultural - O hospital e o CTI, serão descritos alguns aspectos administrativos, conceituais e históricos que emergiram dos relatos dos intensivistas.

\section{O hospital}

A instituição onde foi realizado o estudo é um hospital público, universitário de grande porte, com 400 leitos,"podendo ser estendido até 700 leitos".

Por se tratar de um hospital universitário seus objetivos são a assistência, o ensino e a pesquisa e incorpora uma grande diversidade de profis sionais,"desde um PhD até um indivíduo semianalfabeto".

O hospital é referência para todo o Estado de Minas Gerais e atende a uma clientela bastante diversificada. Sob o ponto de vista dos recursos materiais, "dispõe de uma tecnologia de ponta convivendo com uma profunda obsolescência"."O hospital tem construção vertical, monobloco com 10 andares que abrangem as unidades de internação e unidades administrativas. Além disso, compreende vários anexos, constituídos, na sua maioria, por ambulatórios de várias especialidades. Dessa forma, é uma coisa muito grande e complexa.

No momento de coleta de dados, o hospital estava vivenciando uma crise administrativa, principalmente no que se refere ao quantitativo do pessoal. O hospital tinha em torno de 2000 trabalhadores de enfermagem no seu quadro, dos quais 867 eram celetistas que vinham sendo contratados desde 1987, com o objetivo de suprir o defasado quadro de pessoal e não reduzir, ainda mais, o número de leitos em funcionamento. A partir do ano de 1994, com o remanejamento das vagas do extinto INAMPS, foram alocadas no hospital 903 vagas distribuídas em 42 categorias profissionais, com o objetivo de substituir os profissionais celetistas contratados pela CLT, que deveriam, então, ser demitidos.

Em novembro de 1994, foi realizado concurso público para o preenchimento destas vagas e, em dezembro do mesmo ano, foi publicada a nomeação dos aprovados.

O processo de demissões teve início em janeiro de 1995, em grande defasagem com as admissões, não só sob o ponto de vista quantitativo, como qualitativo.

Em grande parte dos relatos pude identificar revolta, descontentamento, angústia, conflito, insatisfação e desestímulo, relativos ao horário de trabalho, salário, setor de trabalho, falta de pessoal e divergências de natureza política, como mostram os relatos a seguir:

$\mathrm{Na}$ análise dos relatos dos informantes emergiu o domínio cultural: Atributos do hospital, constituído de termos que caracterizam o hospital sob diferentes aspectos, organizados na Taxonomia $\mathrm{n}^{\circ} 1$ : atributos do hospital. 
TAXONOMIA $\mathbf{N}^{\circ}$ 1- ATRIBUTOS DO HOSPITAL

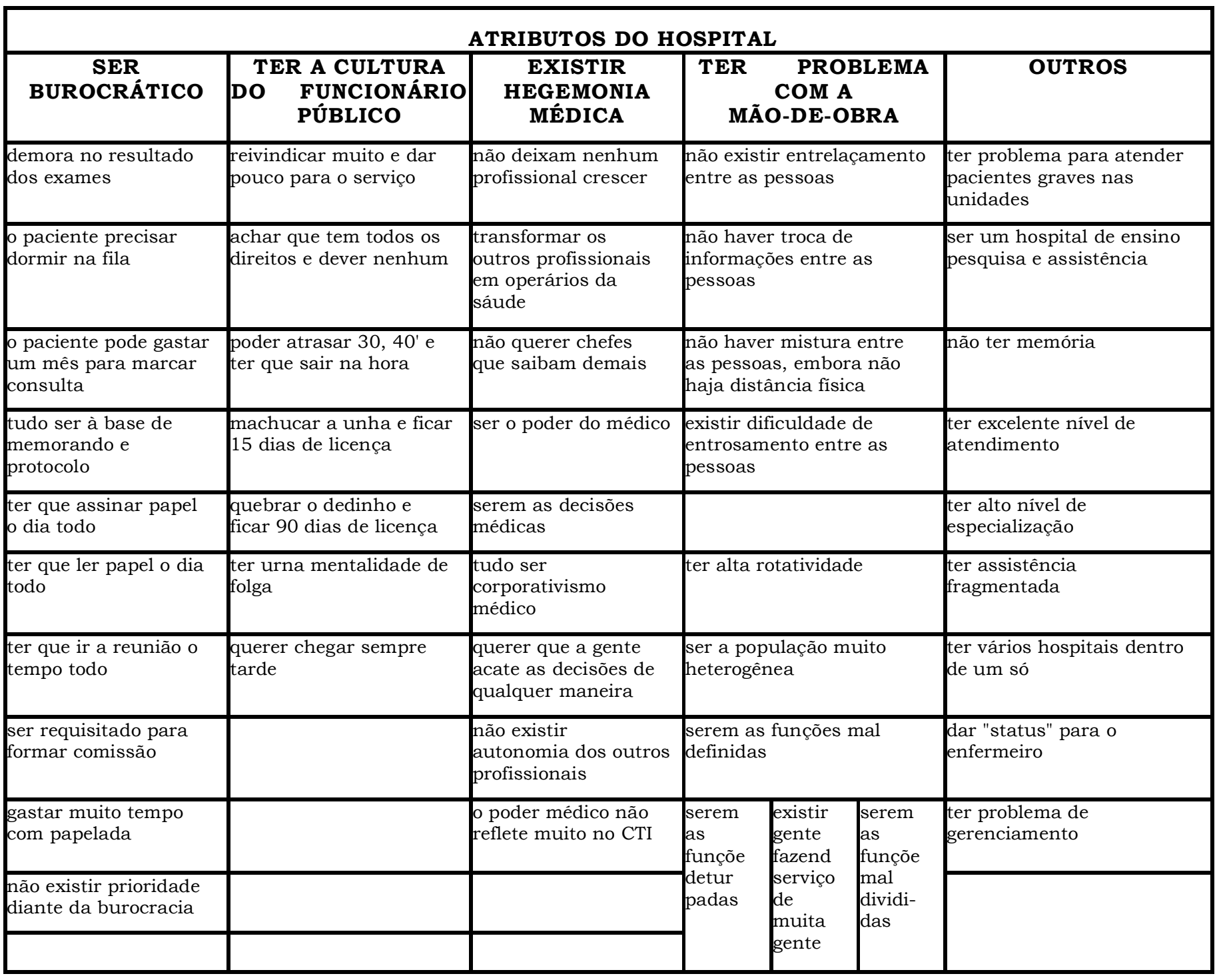

Como pode ser observado na Taxonomia 1, uma das principais características culturais do hospital é "ser burocrático", como se pode identificar no relato abaixo:

"Você recebe alguma coisa...Tudo é à base de memorando. Você vai pedir um sangue com urgência para o paciente, você tem que ir lá primeiro, pegar o papelzinho, escrever registro, ala sul, norte isso $e$ aquilo. Você tem que mandar porque se você não mandar esse papel, não vem sangue".

Os informantes, também indicaram como atributos do Hospital, "ser um bom lugar para trabalhar", não ter memória, ter problema de gerenciamento na área de custeio, conferir "status" ao enfermeiro, existir diferentes subculturas e um alto nível de especialização e fragmentação da assistência, embora o nível de atendimento no Hospital seja considerado "excelente".

"É um dos melhores lugares que já trabalhei. $O$ nivel de atendimento é excelente".

"Pra gente, enquanto enfermeira, dá "status", a nivel salarial não é bom (...)".

"Você tem que cada unidade é um setor, vamos dizer, um mini-hospital dentro do Hospital".

"Tem que ver o coração, mas tem que ver o individuo como um todo, desde o dedão do pé até o último fio do cabelo. Avaliar tudo, tudo. Juntar o indivíduo e não desmembrá-lo em partes, não". 
"Nós estamos vendo agora neste momento as dificuldades na área de custeio, de gerenciamento. O modelo gestor do hospital é um modelo muito inadequado, muito rigido, pesado burocratizado. Ele pode até ser viável em outras estruturas universitárias, mas o hospital é uma coisa muito complexa, precisa de decisões ágeis e esse modelo gestor impede muito as tomadas de decisões, dificulta muito a politica de alocação de recursos, de reequipar o hospital".

O atributo cultural do hospital que aparece de uma forma mais marcante nos relatos dos informantes está relacionado à existência de uma hegemonia médica.

"Aqui tudo é corporativismo médico. Aqui médico pode tudo, médico ganha tudo, leva tudo, manda em tudo".

"Não, mas as coisas precisam ser conversadas. Tem pessoal pra isso? Tem material? Você entendeu? E como se fosse assim, eles tomavam as decisões e queriam que a gente acatasse de qualquer maneira".

Algumas características relativas às pessoas que trabalham no Hospital foram classificadas no atributo "ter a cultura do funcionário público", que, também, emergiu dos relatos dos informantes, como pode ser observado no relato a seguir:

"Eu acho que esta questão seria que as pessoas reivindicam muito mas dão pouco para o serviço. Eu digo na questão do compromisso. Você tem todos os direitos, mas não tem basicamente nenhum dever para com o Hospital. Isso pra mim é a cultura do funcionário público".

Embora "ter a cultura do funcionário público" seja uma característica muita citada pelos informantes, houve uma grande preocupação entre eles em garantir que "era uma minoria". "Eles tinham esse vicio, mas não eram todos não".

Outras características referentes ao pessoal que trabalha no hospital foram agrupadas no atributo "ter problema com a mão-de-obra" (Taxonomia $\mathrm{n}^{\circ} 1$ ). Os problemas citados referiam-se à dificuldade de relacionamento, à heterogeneidade de categorias profissionais, à alta rotatividade e à não definição de papéis:

"A gente tem uma rotatividade muito grande".
"Você vê isso muito claramente. As pessoas não se entrelaçam, não trocam informação. Você não tem uma distância fisica, nem onde termina nem onde começa um setor, mas você vê que as pessoas não se misturam. Se você precisar fazer um remanejamento de uma ala pra outra, você tem dificuldade".

"A enfermeira fica fazendo a função de um tanto de coisa. Então as coisas não eram bem definidas, as tarefas".

\section{O CTI}

Segundo os relatos de antigos membros daquela cultura e dados, também identificados em documento da Organização Pan-Americana de Saúde o ambicioso projeto de desenvolvimento da "atenção progressiva dos hospitais de ensino da América Latina" iniciou-se em 1968, numa integração entre a OPAS e a Fundação Kellogg de Battle Creek, Michigan, com a contribuição técnica do Hospital da Universidade de Michigan, em Ann Arbor. O referido projeto tinha os seguintes objetivos:

"1- Criação de unidades de cuidado intensivo, destinadas ao tratamento de enfermos agudos graves com possibilidade de recuperação, concentrando recursos técnicos e administrativos com vistas à prestação de uma atenção permanente e continua;

2- Organização do cuidado intermediário para pacientes agudos como um modelo a ser instituido nos demais serviços de hospitalização;

3-Estabelecimento de critérios para o aperfeiçoamento dos serviços de consulta externa;

4-Fortalecimento da administração em cada hospital, mediante o aperfeiçoamento do serviço de administração das unidades de cuidado intensivo e sua possivel expansão a outras unidades;

5-Melhoramento dos serviços gerais de apoio, especialmente os de radiologia, laboratório clínico, imunotransfusão, farmácia, central de nutrição e dietética, registros clínicos, estatística e manutenção"(13).

O CTI do Hospital das Clinicas de Belo Horizonte foi inaugurado em 28 de julho de 1969 em decorrência de um convênio firmado entre a OPAS e o Ministério de Saúde representado pela Reitoria da Universidade Federal de Minas Gerais e sua Faculdade de Medicina.

"Naquela época, não existia CTI em Belo Horizonte, talvez existisse em São Paulo, mas não nos moldes da Organização Panamericana de Saúde". 
Embora o CTI tenha surgido com o modelo de Cuidado Progressivo ao Paciente (CPP), o Hospital das Clínicas iniciou pela instalação do CTI, e posteriormente organizou os outros níveis de atendimento, ou seja, o cuidado intermediário e básico.

Segundo documento encontrado nos arquivos do setor, o CTI tinha as seguintes finalidades:

"a- Tratamento de doentes críticos potencialmente recuperáveis; b- Ensino e treinamento de pós-graduação para médicos e enfermeiros e c- Pesquisa científica."

O setor contava com 13 leitos, sendo quatro para "pacientes coronários", oito para "casos clínicos e cirúrgicos" e um leito "isolado".

A equipe médica era constituída de um Comitê Assessor, um médico coordenador, médicos chefes de plantão, médicos assistentes, médicos consultantes e médicos residentes.

A equipe de enfermagem era constituída por uma enfermeira, auxiliares de enfermagem e uma enfermeira chefe, designada pela chefia do serviço de enfermagem, tendo as seguintes atribuições:

"Definir/Manter normas de cuidados de enfermagem, avaliar a necessidade total do paciente; fazer planos para atendê-lo; ensinar - supervisionar prioridades (cuidados de enfermagem); planificar o ensino; cooperar com os médicos; cooperar com a administração; definir necessidades - especificar material de enfermagem; cuidado direto ao paciente".
Antes da inauguração do CTI, houve uma fase de treinamento, ministrado por uma enfermeira, um administrador e um médico da OPAS, abrangendo os três setores de atividades do CTI (enfermagem, administrativo e médico), com o compromisso de prestar assistência à equipe do CTI num período de quatro anos.

A Fundação Kellogg financiou e doou os equipamentos que eram considerados"o básico naquela época" para uma terapia intensiva: os monitores, o carro de ressuscitação e os respiradores.

A planta fisica foi adaptada para que, dentro do possivel, pudesse atender aos moldes preconizados pela OPAS.

Os pacientes atendidos no CTI deveriam preencher os critérios de admissão estabelecidos pelo Comitê Assessor, ou seja, ter instabilidade grave de um sistema fisiológico importante (circulatória, respiratória, metabólica) ou ter a possibilidade de vir a desenvolvê-la. As doenças crônicas em fase terminal e os problemas psiquiátricos graves eram considerados condições que impediam o ingresso dos pacientes no CTI.

É importante registrar que, de acordo com os relatos dos informantes, o CTI representou um marco no desenvolvimento do hospital, em geral, e na enfermagem em particular. Os resultados da criação do CTI estão relacionados na Taxonomia $n .{ }^{\circ} 2$.

TAXONOMIA N. 2 - RESULTADOS DA CRIAÇÃO DO CTI

\begin{tabular}{|c|c|c|}
\hline \multicolumn{3}{|c|}{ RESULTADOS DA CRIAÇÃO DO CTI } \\
\hline ENFERMAGEM & MEDICINA & HOSPITAL \\
\hline exigência de pelo menos um enfermeiro por turno de trabalho & $\begin{array}{l}\text { desenvolvimento do senso de } \\
\text { critério para procedimentos } \\
\text { invasivos }\end{array}$ & $\begin{array}{c}\text { desenvolvímento dos setores de } \\
\text { apoio }\end{array}$ \\
\hline inovações tecnológicas na assistência de enfermagem & & $\begin{array}{l}\text { necessidade de suportes } \\
\text { diagnósticos nas } 24 \text { horas }\end{array}$ \\
\hline $\begin{array}{l}\text { nova jornada de trabalho, com a introdução da modalidade "uma } \\
\text { noite sim, duas não" }\end{array}$ & $\begin{array}{l}\text { desenvolvimento do sentido de } \\
\text { observação clinica }\end{array}$ & $\begin{array}{l}\text { criação do cargo de funcionário } \\
\text { adminístrativo }\end{array}$ \\
\hline relação de um funcionário para cada paciente & \multirow{5}{*}{$\begin{array}{l}\text { introdução de um raciocínio de } \\
\text { clíníca diferente }\end{array}$} & \\
\hline uma nova relação enfermagem/paciente & & \\
\hline consolidação da função de enfermeiro assistencial & & \\
\hline introdução da elaboração do plano de cuidados & & \\
\hline liberação da enfermagem de várias atribuições burocráticas & & \\
\hline \multicolumn{2}{|c|}{ consolidação do CTI como órgão formador de recursos humanos } & \\
\hline \multicolumn{2}{|c|}{ assístência permanente e contínua nas 24 horas } & \\
\hline & tência mediada pela tecnologi & \\
\hline
\end{tabular}


Desta forma, assim como explica uma informante, o CTI"puxou o desenvolvimento da enfermagem e do Hospital". A sua criação acarretou mudanças com relação à assistência em geral, e de enfermagem, em particular. A assistência de enfermagem em CTI, visto que contínua nas 24 horas, envolvendo procedimentos de alta complexidade, com alto grau de incorporação tecnológica, passou a exigir planejamento, melhoria dos profissionais em termos quantitativos e qualitativos. Inicia-se, no CTI, a prática de elaboração de plano de cuidados, a assistência direta ao paciente pelo enfermeiro, consolidando a função do enfermeiro assistencial e exigência da presença deste profissional em todos os turnos de trabalho.

A exigência da atuação do enfermeiro numa assistência e procedimentos cada vez mais complexos aproxima-o do paciente, levando à criação do cargo de "funcionário administrativo", que passou a assumir as funções ditas burocráticas.

A assistência de enfermagem e médica passaram a ser mediadas pela tecnologia de uma forma mais evidente, tendo em vista a significativa incorporação de inovações tecnológicas na prática dos profissionais intensivistas.

O hospital, da mesma forma, passa por transformações importantes frente as novas exigências de uma assistência continua nas 24 horas do dia. Tais exigências acarretam o desenvolvimento dos setores de apoio, tendo em vista a necessidade de suporte diagnóstico nas 24 horas.

Nos relatos abaixo estão relacionados alguns dos resultados da criação do CTI, segundo os informantes:

"Nós começamos do nada e foi a base de tudo, foi a base dos outros. Foi uma época que marcou um progresso do Hospital da clínicas, uma evolução e eu acho que foi importante".

"O CTI puxou o desenvolvimento da enfermagem muito grande.. A gente dava assistência de 24 horas, era muito bem seguido, não deixava passar nada".

"O enfermeiro assistencial, lá fora devia ter, mas em número menor".

"Os laboratórios foram melhorando, introduziram coisas que não tinham e que passaram a ter por causa do CTI. Porque a gente não podia deixar de ter um Raio $X$ nas 24 horas...".
"Eu falei muito em defasagem tecnológica. Eu acho que o grande mérito do CTI desse hospital, pelo fato da gente nunca ter contado com muita fartura tecnológica, foi de desenvolver no médico o sentido de observação clínica muito grande, um senso de critério pra indicação dessas medidas invasivas e utilização de tecnologia".

Ao descrever o setor na atualidade, os informantes apontam a sua descaracterização, enquanto CTI, devido a uma perda de referências profissionais e a uma ruptura com valores de um CTI considerado"modelo", num passado muito recente, mas com um pequeno número de pessoas capazes de transmitir esses valores para"essa nova geração que está chegando".

Finalmente, os informantes descreveram os significados da terapia intensiva. Esses significados incorporam a dimensão tecnológica (serviço de vanguarda, método terapêutico, formador de recursos humanos), o sentido de unidade (equipe fazendo alguma coisa junto), bem como a importância conferida à assistência de enfermagem contínua, entre outros (é a enfermagem, é desafio).

"O CTI continuou sendo um grande formador de recursos humanos. Eu acredito que grande parte dos médicos hoje que exercem terapia intensiva em $\mathrm{BH}$ tenham feito estágio aqui no CTI".

"É uma questão de gostar de CTI, gostar de emergência. Se você quer desafio... É para mim um desafio e para a maioria das pessoas também é".

"É expressiva, é importantíssima. Não que eu esteja desfazendo da classe médica, mas eu acho que a enfermagem, ela é tudo dentro do CTI, 24 horas ali do lado".

"Sendo método terapêutico tem que ter indicações, tem contra indicações, tem efeitos colaterais importantes".

\section{CONCLUSÃO}

$\mathrm{Na}$ contextualização do cenário cultural, considerei importante caracterizar, inicialmente o hospital onde foi realizado o estudo. Embora já tivesse tomado essa decisão por ocasião da elaboração do projeto, a idéia inicial era que essa contextualização 
fosse resgatada de documentos formais tais como, regimentos, manuais, entre outros. Na verdade isso também ocorreu.

Ao realizar a pesquisa, foram emergindo domínios e taxonomias dos relatos dos informantes que se referiam ao hospital, embora não fosse este o objetivo primeiro do estudo. Diante disso e, coerente com as premissas deste trabalho, optei por caracterizar o hospital sob o ponto de vista oficial, mas também e principalmente, sob o ponto de vista daquelas pessoas que vivenciam o cotidiano da instituição.

Assim, na caracterização do hospital, estão mescladas características referidas pelos informantes,

\section{REFERÊNCIAS BIBLIOGRÁFICAS}

1. QUIJANO PITMAN, F; QUIJANO ORVAÑANOS, F. História de las unidades de cuidados intensivos: dos antecedentes mexicanos. Gac Med Méx, 1991. $127(4): 381-4$.

2. FAIRMAN, J. Watchful vigilance, nursing care, technology, and the development of intensive care units. Nurs Res, 1992; 41(1):56-60, 1992.

3. CARNEIRO, AM. Comunicação enfermeiro-paciente na UTI: estudo interativo do processo de comunicação. (dissertação) Rio de Janeiro (RJ): Escola de Enfermagem Ana Nerí/UFRJ;1982.

4. LOPÉZ, M. Centro de tratamento intensivo. Rev Assoc Méd Minas Gerais 1971;22(4):211-16.

5. HENDERSON, A. The sígnificance for critical care nurses of the "dying with dignity" legislation. Aust Crit Care 1994; (2): 23-6.

6. HENDERSON, JN. The culture of special care unit: an anthropological perspective on ethnographic research in nursing home settings. Alzheimer Dis Assoc Disord, 1994; 8 Supp1 1:5410-6.

7. ROSENAL, TW et al. Support for information management in critical care: a new approach to identify needs. Proc Annu Symp Comput Appl Med Care 1995, p.2-6.

8. RAINES, D. Deciding what to do when the patient can't speak: a preliminary analysis of ethnographic study of professional nurses in the neonatal intensive care unit. Neonatal Netw 1993;12(6):43-8. outras decorrentes das observações da pesquisadora, como também alguns dados gerais coletados em documentos oficiais, sendo os limites dessas perspectivas, algumas vezes, tênues.

Neste estudo, foram utilizadas as técnicas de coleta de dados aderentes ao método etnográfico para descrição do cenário cultural no qual foi realizado o estudo. Os dados aqui apresentados demonstram que a etnografia se constitui numa estratégia metodológica adequada para contextualização do cenário cultural do CTI, sob o ponto de vista de quem vivência o cotidiano do trabalho na terapia intensiva, constituindo-se em parte fundamental do conhecimento cultural de seus membros.

9. Chase, SK. The social context of critical care clinical judgment. Heart Lung 1995; 24(2):154-62.

10. Peixoto, MRB. A prioridade, o isolamento e as emoções: estudo etnográfico do processo de socialização em um centro de tratamento intensivo. (tese) São Paulo (SP): Escola de Enfermagem/USP;1996.

11. Gregory, KL. Native-view paradigms; multiple cultures and culture conflicts in organizations. Adm Sci Q 1983; 28: $359-76$.

12. SPRADLEY, J. The ethnographic interview. New York: Holt Rinehart \& Winston; 1979.

13. Organízação Panamericana de la Salud. Unidades de cuidado Intensivo para la America Latina. Washington; 1973. (OPAS - Publicación científica 264).
Artigo recebido em 22/02/00

Artigo aprovado em 23/07/01 